\title{
DETERMINANTS IN PROJECTIVE MODULES ${ }^{1)}$
}

\author{
OSCAR GOLDMAN
}

\section{Introduction}

The definition of the determinant of an endomorphism of a free module depends on the following fact: If $F$ is a free $R$-module of rank $n$, then the homogeneous component $\wedge^{n} F$, of degree $n$, of the exterior algebra $\wedge F$ of $F$ is a free $R$-module of rank one. If $\alpha$ is an endomorphism of $F$, then $\alpha$ extends to an endomorphism of $\wedge F$ which in $\wedge{ }^{n} F$ is therefore multiplication by an element of $R$. That factor is then defined to be the determinant of $\alpha$. (A discussion of this theory may be found in [4].)

This procedure cannot be applied in general to finitely generated projective modules since, for such modules, it may happen that no homogeneous component of the exterior algebra is free of rank one.

In this note we show how to define the determinant of an endomorphism of a finitely generated projective module over any commutative ring. If $E$ is a finitely generated projective $R$-module, then $E$ is a direct summand of a finitely generated free module: $E+E_{1}=F$. If $\alpha$ is an endomorphism of $E$, extend $\alpha$ to an endomorphism $\alpha_{1}$ of $F$ by defining $\alpha_{1}$ to be the identity on $E_{1}$. It is proved that det $\alpha_{1}$ depends only on $E$ and $\alpha$, and not on the choice of $E_{1}$; we then $\operatorname{define} \operatorname{det} \alpha$ to be $\operatorname{det} \alpha_{1}$. The usual elementary properties of the determinant are valid in this more general setting and are immediate consequences of the definition.

By forming the $R[X]$-module $E \otimes R[X]$, we define the characteristic polynomial $\varphi(\alpha, E, X)$ of an endomorphism $\alpha$ of $E$ as $\operatorname{det}(X-\alpha \otimes 1)$. The CayleyHamilton theorem, to the effect that $\varphi(\alpha, E, X)$ is in the kernel of the homomorphism $R[X] \rightarrow \operatorname{Hom}_{R}(E, E)$ defined by $X \rightarrow \alpha$, is readily verified.

The characteristic polynomial $\varphi(0, E, X)$ of the zero endomorphism has a number of interesting properties. If $E$ is free of rank $n$, then certainly

Received October 31, 1960.

1) This work was performed under a grant from the National Science Foundation. 
$\varphi(0 . E, X)=X^{n}$, but this is not the case in general. Here one finds $\varphi(0, E, X)=$

$\sum_{i=0}^{n} e_{i} X^{i}$ with the $e_{i}$ mutually orthogonal idempotents whose sum is 1 ; and every polynomial of this type can occur. The coefficients have the following interpretation: $e_{0}$ generates the annihilator of $E$, while $e_{0}+\cdots+e_{i}$ generates the annihilator of $\wedge^{i+1} E$. Also, for a given $E$ all the polynomials $\varphi(\alpha, E, X)$ have the same degree and the same leading coefficient independently of $\alpha$.

If $\mathfrak{p}$ is a prime ideal of $R$, the $R_{\mathfrak{p}}$-module $E \otimes R_{\mathfrak{p}}$, being a finitely generated projective module over a local ring, is free; we call its rank the p-rank of $E$. With the above notation $\varphi(0, E, X)=\sum_{i=1}^{n} e_{i} X^{i}$, we have the following result: given $\mathfrak{p}$, exactly one coefficient fails to lie in $\mathfrak{p}$, and if $e_{i} \notin \mathfrak{p}$, then the $\mathfrak{p}$-rank of $E$ is $i$.

We follow the customary terminology in dealing with projective modules: all rings have unit elements, ring homomorphisms carry the units into units and all modules are unitary.

\section{Section 1. The Determinant of an Endomorphism}

Let $R$ be a commutative ring and $E$ a finitely generated projective $R$-module. Because $E$ is both projective and finitely generated, $E$ is a direct summand of a finitely generated free $R$-module: $F_{1}=E+E_{1}$. If $\alpha: E \rightarrow E$ is an endomorphism of $E$, we extend $\alpha$ to an endomorphism $\alpha_{1}$ of $F_{1}$ by $\alpha_{1}=\alpha+1$, that is, $\alpha_{1}$ is the identity map of $E_{1}$. If should be noticed that if both $E$ and $E_{1}$ are free $R$-modules, then $\alpha$ and $\alpha_{1}$ have the same determinant. Therefore it would be reasonable to define in general the determinant of $\alpha$ as $\operatorname{det} \alpha_{1}$. This is possible once we verify that $\operatorname{det} \alpha_{1}$ does not depend on the particular representation of $E$ as a direct summand of a free module. In order to show that this is the case, we need the following lemma.

Leмma 1.1. Let $R$ be a commutative ring, $A$ an $R$-module. If $\mathfrak{p}$ is a prime ideal of $R$, let $f_{\mathfrak{p}}: A \rightarrow A \otimes{ }_{R} R_{\mathfrak{p}}$ be defined by $f_{\mathfrak{p}}(a)=a \otimes 1$. If $a \in A$ is such that $f_{\mathfrak{m}}(a)=0$ for all maximal ideals $m$ of $R$, then $a=0$.

Proof. It follows directly from the definition of the ring of quotients $R_{\mathrm{p}}$ that an element $a \in A$ is in the kernel of $f_{\mathfrak{p}}$ if, and only if, there exists an element $c \in R$ with $c \notin p$ and $c a=0$. If $a \in A$, let $\Re$ be the annihilator of $a$ in $R$. If $f_{\mathfrak{m}}(a)=0$ for all maximal ideals $\mathfrak{m}$, then $\mathfrak{R} \neq \mathfrak{m}$, for any maximal ideal. 
Consequently, $\Re=R$ and $a=0$.

As an immediate consequence of the lemma we have the following proposition.

Proposition 1.2.' Let $E$ be a finitely generated projective R-module. For $i=1,2$ let $F_{i}=E+E_{i}$ be a finitely generated free $R$-module. If $\alpha \in \operatorname{Hom}_{R}(E, E)$, let $\alpha_{i} \in \operatorname{Hom}_{R}\left(F_{i}, F_{i}\right)$ be defined by $\alpha_{i}=\alpha \dot{+}$. Then $\operatorname{det} \alpha_{1}=\operatorname{det} \alpha_{2}$.

Proof. Let $\mathrm{m}$ be a maximal ideal of $R$. The ring of quotients $R_{\mathfrak{l}}$ is a local ring (not necessarily noetherian) so that $E \otimes R_{\mathfrak{m}}, E_{i} \otimes R_{\mathfrak{l t}}$ are all free $R_{\mathfrak{m}}$-modules. (It follows from prop. 5.1, ch. VIII of [3] that a finitely generated projective module over a local ring is free). Then, tensor with $R_{\mathfrak{m}}$ the direct sum $F_{1}=E+E_{1}$ to give $F_{1} \otimes R_{\mathfrak{m}}=E \otimes R_{\mathfrak{m}}+E_{1} \otimes R_{\mathfrak{m}}$. The endomorphisms $\alpha$ and $\alpha_{1}$ extend to endomorphisms $\alpha \otimes 1$ and $\alpha_{1} \otimes 1$ which have the same relation as do $\alpha$ and $\alpha_{1}$. If $f_{\mathfrak{m}}: R \rightarrow R_{\mathfrak{m}}$ is the canonical map, it is clear that $\operatorname{det}\left(\alpha_{1} \otimes 1\right)$ $=f_{\mathfrak{m}}\left(\operatorname{det} \alpha_{1}\right)$, while at the same time $\operatorname{det}\left(\alpha_{1} \otimes 1\right)=\operatorname{det}(\alpha \otimes 1)$. Now, the same situation prevails in $F_{2}$, so that $f_{\mathfrak{m}}\left(\operatorname{det} \alpha_{1}\right)=\operatorname{det}(\alpha \otimes 1)=f_{\mathfrak{m}}\left(\operatorname{det} \alpha_{2}\right)$, and this is so for all maximal ideals $m$ of $R$. Applying the lemma with $A=R$ shows that $\operatorname{det} \alpha_{1}=\operatorname{det} \alpha_{2}$.

In view of this proposition, we define $\operatorname{det} \alpha_{1}$ as the determinant of $\alpha$, and denote it by $\operatorname{det} \alpha$. It is clear that for a free module the present definition coincides with the usual one.

There are several properties of determinants which follow directly from the definition.

Proposition 1.3. Let $E$ be a finitely generated projective R.module. If $\alpha, \beta \in \operatorname{Hom}_{R}(E, E)$, then $\operatorname{det}(\alpha \beta)=\operatorname{det} \alpha \operatorname{det} \beta . A$ Also, $\operatorname{det} 1=1$. Finally, $\alpha$ is an automorphism of $E$ if, and only if, $\operatorname{det} \alpha$ is a unit in $R$.

Proof. We use the same notation as above, $F=E+E_{1}$. It is clear that $(\alpha \beta)_{1}=\alpha_{1} \beta_{1}$, from which the multiplicative property of det follows. Also $1_{1}=1$, whence $\operatorname{det} 1=1$. If $\alpha$ is an automorphism of $E$, these relations show that $\operatorname{det} \alpha$ is a unit in $R$. On the other hand, suppose that $\operatorname{det} \alpha$ is a unit in $R$. Then $\alpha_{1}$ is an automorphism of $F$. One verifies immediately that $\alpha_{1}^{-1}$ maps $E$ into itself, and is the inverse of $\alpha$ on $E$. Therefore $\alpha$ is an automorphism of $E$.

2) I am indebted to Maurice Auslander for suggesting the main idea of this proof. 
Suppose that $f: R \rightarrow S$ is a homomorphism. Then $f$ gives $S$ the structure of an $R$-module and $E \otimes{ }_{R} S$ is a finitely generated projective $S$-module, where $E$ is a finitely generated projective $R$-module. If $\alpha \in \operatorname{Hom}_{R}(E, E)$, then $\alpha \otimes 1$ is an endomorphism of $E \otimes S$ over $S$. In case $E$ is $R$-free, it is obvious that $\operatorname{det}(\alpha \otimes 1)=f(\operatorname{det} \alpha)$. If follows immediately from the definition of determinant that this relation holds also in the case where $E$ is projective, not necessarily free. Thus, we have:

Proposition 1.4. Let $E$ be a finitely generated projective $R$-module and $\alpha \in \operatorname{Hom}_{R}(E, E)$. Let $f: R \rightarrow S$ be a ring homomorphism. Then, $\alpha \otimes 1 \in \operatorname{Hom}_{S}$ $(E \otimes S, E \otimes S)$ and $\operatorname{det}(\alpha \otimes 1)=f(\operatorname{det} \alpha)$.

There is a final simple property of determinants analogous to the classical situation. We shall omit the proof since it is a straightforward consequence of the definition.

Proposition 1.5. Let $E_{1}$ and $E_{2}$ be finitely generated projective $R$-modules, and let $\alpha_{i} \in \operatorname{Hom}_{R}\left(E_{i}, E_{i}\right)$. Then, $\operatorname{det}\left(\alpha_{1} \dot{+} \alpha_{2}\right)=\operatorname{det} \alpha_{1} \operatorname{det} \alpha_{2}$.

\section{Section 2. The Characteristic Polynomial}

Let $R$ be as above, and let $R[X]$ be the ring of polynomials in one indeterminate over $R$. If $E$ is a finitely generated projective $R$-module, then $E \otimes R[X]$ is a finitely generated projective $R[X]$-module. Let $\alpha \in \operatorname{Hom}_{R}(E, E)$. Then $\alpha \otimes 1$ is an endomorphism of $E \otimes R[X]$, while multiplication by $X$ is another endomorphism. Thus, $X-\alpha \otimes 1 \in \operatorname{Hom}_{R[X]}(E \otimes R[X], E \otimes R[X])$ and $\operatorname{det}(X-\alpha \otimes 1) \in R[X]$ is called the characteristic polynomial of $\alpha$. We shall denote it by $\varphi(\alpha, E, X)$. It follows immediately from proposition 1.5 that $\varphi\left(\alpha_{1}+\alpha_{2}, E_{1}+E_{2}, \mathrm{X}\right)=\varphi\left(\alpha_{1}, E_{1}, X\right) \varphi\left(\alpha_{2}, E_{2}, X\right)$. Also if $f: R \rightarrow S$ is a ring homomorphism, then $\varphi(\alpha \otimes 1, E \otimes S, X)=f^{\prime} \varphi(\alpha, E, X)$, where $f^{\prime}: R[X] \rightarrow S[X]$ is the natural extension of $f$.

Theorem 2.1. (CAyley-Hamilton). $\varphi(\alpha, E, X)$ is in the kernel of the homomorphism $R[X] \rightarrow \operatorname{Hom}_{R}(E, E)$ defined by $X \rightarrow \alpha$.

Proof. We note first that the Cayley-Hamilton theorem is true in case $E$. is free. The usual proof of the theorem for vector spaces, as for example in [2], is valid for free modules over any commutative ring. 
Now, let $E$ be projective and $\alpha \in \operatorname{Hom}_{R}(E, E)$. Let $\omega$ be the image of $\varphi(\alpha, E, X)$ under the homomorphism $X \rightarrow \alpha$. We wish to show that $\omega=0$. To do so, we consider the submodule $\omega(E)$ of $E$. If $m$ is any maximal ideal of $R$, all objects under consideration localize properly, and $\omega(E) \otimes R_{\mathfrak{m}}=0$ because $E \otimes R_{\mathfrak{m}}$ is a free $R_{\mathfrak{m}}$-module. It follows from lemma 1.1 that $\omega(E)=0$, that is, $\omega=0$.

The characteristic polynomial of the zero endomorphism is especially interesting. Clearly $\varphi(0, E, X)$ is the determinant, in $R[X]$, of the endomorphism defined by multiplication by $X$ in $E \otimes R[X]$. Using the homomorphism $R[X]$ $\rightarrow R$ defined by $X \rightarrow 1$ shows that $\varphi(0, E, 1)=1$. The multiplicative property of the determinant gives $\varphi(0, E, X Y)=\varphi(0, E, X) \varphi(0, E, Y)$ where $X$ and $Y$ are independent indeterminates over $R$. Set $\varphi(0, E, X)=\sum_{i=0}^{n} e_{i} X_{i}, e_{i} \in R$. Applying the multiplicative property shows that $\sum_{i, j} e_{i} e_{j} X^{i} Y^{j}=\sum_{k} e_{k} X^{k} Y^{k}$, so that comparing the coefficients of the different monomials gives $e_{i} e_{j}=\delta_{i j} e_{i}$. The equation $\varphi(0, E, 1)=1$ translates into $\sum e_{i}=1$. Thus, we have proved:

Proposition 2.2. If $\varphi(0, E, X)=\sum_{i=0}^{n} e_{i} X^{i}$ is the characteristic polynomial of the zero endomorphism of $E$, then the $e_{0}, e_{1}, \ldots, e_{n}$ are mutually orthogonal idempotents with $e_{0}+e_{1}+\cdots e_{n}=1$.

Of course, if $E$ is a free $R$-module of rank $n$, then $\varphi(0, E, X)=X^{n}$. In general the polynomial $\varphi(0, E, X)$ need not have that form.

Proposition 2.3. Let $e_{0}, \ldots, e_{n}$ be mutually orthogonal idempotents in $R$ whose sum is 1 . Then, there exists a finitely generated projective R-module $E$ such that $\varphi(0, E, X)=\sum_{i=0}^{n} e_{i} X^{i}$.

Proof. The conditions imposed on the $e^{\prime} s$ imply the relation $\sum_{i=0}^{n} e_{i} X^{i}=$ $\prod_{j=1}^{n}\left(1-e_{j}+e_{j} X\right)^{j}$, as may be verified by direct computation. Since $\varphi\left(0, E_{1} \dot{+} E_{2}, X\right)$ $=\varphi\left(0, E_{1}, X\right) \varphi\left(0, E_{2}, X\right)$, it follows that we need only prove the following: if $e \in R$ is an idempotent, then there is a finitely generated projective $R$-module $A$ such that $\varphi(0, A, X)=1-e+e X$. Now, since $e$ is idempotent, $A=R e$ is a direct summand of $R$, so that it is finitely generated projective. Furthermore, the definition of determinant shows directly that $\varphi(0, R e, X)=1-e+e X$.

The Cayley-Hamilton theorem applied to $\varphi(0, E, X)$ shows that $\varphi(0, E, 0)$ $=e_{0}$ annihilates $E$. Also, it is clear that $\varphi(0, E, 0)=\operatorname{det} 0$, and the above con- 
struction shows that det 0 need not be 0 in general; although it is the zero endomorphism of $E$. Some properties of $\operatorname{det} 0$ will be discussed later.

\section{Section 3. The Local Rank of a Module}

Let $E$ be a finitely generated projective $R$-module. If $\mathfrak{p}$ is a prime ideal of $R$, then $E \otimes R_{\mathfrak{p}}$ is a free $R_{\mathfrak{p}}$-module of finite rank. We shall call the rank of $E \otimes R_{\mathfrak{p}}$ the p-rank of $E$. It is sometimes of interest to know how the p-rank of $E$ depends on $\mathfrak{p}$.

THEOREM 3.1. Let $\varphi(0, E, X)=\sum_{i=0}^{n} e_{i} X^{i}$ be the characteristic polynomial of the zero endomorphism of $E$. If $\mathfrak{p}$ is a prime ideal of $R$, then exactly one of the $e^{\prime} s$ is not in $\mathfrak{p}$; if $e_{i} \notin \mathfrak{p}$, then the p-rank of $E$ is $i$. If $e_{j} \neq 0$ there is a prime ideal $p$ such that the $p$-rank of $E$ is $j$.

Proof. Since $\sum_{i=0}^{n} e_{i}=1$, not all of the $e^{\prime} s$ are in $p$; suppose $e_{i} \notin p$. For $j \neq i$ we have $e_{i} e_{j}=0$, so that $e_{j} \in \mathfrak{p}$ because $\mathfrak{p}$ is a prime ideal. Since $e_{i} \notin \mathfrak{p}$ and $e_{i} e_{j}=0$ for $j \neq i$, it follows that $f_{\mathfrak{p}}\left(e_{j}\right)=0$ where $f_{\mathfrak{p}}: R \rightarrow R_{\mathfrak{p}}$. Also, because $e_{i}\left(1-e_{i}\right)=0$, we have $f_{\mathfrak{p}}\left(e_{i}\right)=1$. Therefore if $f_{\mathfrak{p}}^{\prime}: R[X] \rightarrow R_{\mathfrak{p}}[X]$ is the extension of $f_{\mathfrak{p}}$, we find that $f_{\mathfrak{p}}^{\prime}(0, E, X)=X^{i}$. But $f_{\mathfrak{p}}^{\prime}(0, E, X)=\varphi\left(0, E \otimes R_{\mathfrak{p}}, X\right)$, while $\varphi\left(0, E \otimes R_{\mathfrak{p}} . X\right)=X^{m}$ if $m$ is the p-rank of $E$. Thus, $m=i$.

Now, suppose $e_{j} \neq 0$. Since the intersection of all prime ideals of $R$ is the set of nilpotent elements of $R$, and since no non-zero idempotent is nilpotent, we have $e_{j} \notin \mathfrak{p}$ for some prime ideal $\mathfrak{p}$. It follows from the above that the $\mathfrak{p}$ rank of $E$ is just $j$.

We have as an immediate consequence the following:

Corollary 3.2. If $R$ has no non-trivial idempotents, then the p-rank of $E$ is the same for all $\mathrm{p}$.

If we set $\varphi(0, E, X)=\sum_{i=0}^{n} e_{i} X^{i}$ with $e_{n} \neq 0$, theorem 3.1 shows that $n$ is the maximum of all p-ranks of $E$. We shall call $n$ the R-rank of $E$. Theorem 3.1 also shows that the p-ranks of $E$ are completely determined by the knowledge of the polynomial $\varphi(0, E, X)$.

THEOREM 3.3. If the R-rank of $E$ is $n$, then for any endomorphism $\alpha$ of $E$ the polynomial $\varphi(\alpha, E, X)$ has degree $n$. Furthermore, the leading coefficient of $\varphi(\alpha, E, X)$ is independent of $\alpha$. 
Proof. Set $\varphi(\alpha, E, X)=\sum_{i=0}^{m} a_{i} X^{i}$, with $a_{m} \neq 0$. There exists a prime ideal $p$ such that $f_{\mathfrak{p}}\left(a_{m}\right) \neq 0$, with $f_{\mathfrak{p}}: R \rightarrow R_{\mathfrak{p}}$ as usual. Since $\sum f_{\mathfrak{p}}\left(a_{i}\right) X^{i}$ is the characteristic polynomial of $\alpha \otimes 1$ in $E \otimes R_{\mathfrak{p}}$, its degree $m$ is equal to the p-rank of $E$, which in turn is $\leq n$. Hence $m \leq n$. Since there exists some prime ideal $p$ such that the $p$-rank of $E$ is $n$, it follows that $m \geq n$, hence $m=n$.

Let $\varphi(0, E, X)=\sum_{i=0}^{n} e_{i} X^{i}$. If $\mathfrak{p}$ is a prime ideal such that $e_{n} \notin \mathfrak{p}$, then the $\mathfrak{p}$-rank of $E$ is $n$ and therefore $f_{\mathfrak{p}}\left(a_{n}\right)=1$. Hence $a_{n}-1$ is annihilated by some element of $R$ not in $\mathfrak{p}$. Denote by $\mathfrak{b}$ the annihilator of $a_{n}-1$. We have therefore the implication: $e_{n} \notin \mathfrak{p} \Rightarrow \mathfrak{b} \leftarrow \mathfrak{p}$. It follows immediately that $e_{n} \in \operatorname{rad} \mathfrak{b}$. Since $e_{n}$ is idempotent, this shows that $e_{n} \in \mathfrak{b}$, or $e_{n} a_{n}=e_{n}$.

Now suppose $\mathfrak{p}$ is such that $e_{n} \in \mathfrak{p}$. Then the $\mathfrak{p}$-rank of $E$ is less than $n$, so that $f_{\mathfrak{p}}\left(a_{n}\right)=0$. If $\mathrm{c}$ is the annihilator of $a_{n}$, we find: $e_{n} \in \mathfrak{p} \Rightarrow \mathfrak{c} \mp \mathfrak{p}$. Now, $e_{n} \in \mathfrak{p}$ is the same as $1-e_{n} \notin \mathfrak{p}$, and therefore we conclude that $1-e_{n} \in \operatorname{rad} \mathrm{c}$. Again, because $1-e_{n}$ is idempotent, we have $1-e_{n} \in \mathrm{c}$ or $\left(1-e_{n}\right) a_{n}=0$. Thus, $\boldsymbol{a}_{n}=\boldsymbol{e}_{n} \boldsymbol{a}_{n}$. Since we have already established the equality $\boldsymbol{e}_{n} a_{n}=\boldsymbol{e}_{n}$, we conclude that $a_{n}=e_{n}$.

\section{Section 4. The Exterior Algebra}

Before starting on the proper subject matter of this section, we need some preliminary results.

Lemma 4.1. Let $e$ and $e^{\prime}$ be idempotents in the commutative ring $R$. If $R e=R e^{\prime}$, then $e=e^{\prime}$. If $\operatorname{rad}(R e)=\operatorname{rad}\left(R e^{\prime}\right)$, then $e=e^{\prime}$.

Proof. If $R e=R e^{\prime}$ then $e=a e^{\prime}$ and $e^{\prime}=b e$ with $a$ and $b$ suitable elements of $R$. Combining the equations shows that $e=a b e$, and $e^{\prime}=a b e^{\prime}$. From the idempotence of $e$ and $e^{\prime}$ we get $b^{2} e=e^{\prime}$ or $b e^{\prime}=e^{\prime}$. Multiplying by a yields $a b e^{\prime}=a e^{\prime}=e$, while $a b e^{\prime}=e^{\prime}$ or $e=e^{\prime}$.

From $\operatorname{rad}(R e)=\operatorname{rad}\left(R e^{\prime}\right)$ and the idempotence of $e$ and $e^{\prime}$ we get $R e=R e^{\prime}$ and therefore $e=e^{\prime}$.

If $E$ is an $R$-module we call the annihilator of $E$, and denote by $a(E)$, the set of all $\mathrm{a} \in R$ such that $a E=0$. Clearly $a(E)$ is an ideal in $R$. We say that $E$ is faithful if $a(E)=0$.

Leмма 4.2. If $E$ is a finitely generated projective $R$-module, then a(E) is a direct summand of $R$. 
Proof. The lemma is a consequence of the considerations in the appendix of [1]. However for the sake of completeness we include a proof of the statement. Let $\mathfrak{I}$ be the ideal in $R$ generated by all $f(x)$ as $x$ ranges through $E$ and $f$ ranges through $\operatorname{Hom}_{i}(E, R)$. Then, we shall show that $R=\mathfrak{a}(E) \dot{+} \mathfrak{I}$. If $a \in \mathfrak{a}(E)$, then $a f(x)=f(a x)=0$, so that $\mathfrak{a}(E) \mathfrak{I}=0$.

Let $x_{1}, \ldots, x_{n}$ be a set of generators of $E$, and let $F$ be a free $R$-module of rank $n$. By mapping the generators of $F$ onto the $x_{i}$ we obtain an epimorphism of $F$ on $E$; since $E$ is projective, it follows that $E$ is a direct summand of $F$. Let $f_{1}, \ldots, f_{n}$ be the restrictions to $E$ of the coordinate maps of $F$. Then, $f_{i} \in \operatorname{Hom}_{R}(E, R)$ and $\sum f_{i}(y) x_{i}=y$, for all $y \in E$. In particular, we have $x_{j}=\sum_{i} f_{i}\left(x_{j}\right) x_{i}$ or $\sum_{i}\left(f_{i}\left(x_{j}\right)-\delta_{i j}\right) x_{i}=0$. It follows from this that $\operatorname{det}\left(f_{i}\left(x_{j}\right)-\delta_{i j}\right)$ $\in \mathfrak{a}(E)$. Now, each $f_{i}\left(x_{j}\right) \in \mathfrak{T}$ and the value of $\operatorname{det}\left(f_{i}\left(x_{j}\right)-\delta_{i j}\right)$ is $(-1)^{n}+t$, where $t$ is also in the ideal $\mathfrak{I}$. Thus, $1 \in \mathfrak{a}(E)+\mathfrak{I}$ so that $a(E)+\mathfrak{I}=R$. It follows from this and the fact that $a(E) \mathfrak{I}=0$ that $R$ is the direct sum of $a(E)$ and $\mathfrak{I}$.

If $E$ is an $R$-module we denote by $\wedge E$ the exterior algebra of $E$, and by $\wedge^{i} E$ the homogeneous part of degree $i$ of $\wedge E$. (We refer the reader to [4] for details concerning $\wedge E$ ). In general we have $\wedge^{\circ} E=R$ and $\wedge^{1} E=E$. Also, $\wedge^{i}\left(E_{1} \dot{+} E_{2}\right)=\sum_{j=0}^{i} \wedge^{j} E_{1} \otimes \wedge^{i-j} E_{2}$ (direct sum). If $F$ is a free module, then $\wedge^{i} F$ is also free. If follows from this that $\wedge^{i} E$ is a finitely generated projective $R$-module whenever $E$ is a finitely generated projective $R$-module.

THEOREM 4.3. Let $E$ be a finitely generated projective $R$-module of R-rank $n$ and let $\varphi(0, E, X)=\sum_{i=0}^{n} e_{i} X^{i}$. Then $a\left(\wedge^{i+1} E\right)$ is generated by $e_{0}+e_{1}+\cdots+e_{i}$. In particular $\wedge^{n} E \neq 0$ and $\wedge^{n+1} E=0$. Finally, $\wedge^{n} E$ is a faithful $R$-module if, and only if, $\varphi(0, E, X)=X^{n}$, and in that case all $\wedge^{i} E$ are faithful, for $0 \leq i \leq n$.

Proof. Let $\mathfrak{p}$ be a prime ideal of $R$ and suppose that the $\mathfrak{p}$-rank of $E$ is $m$. Then, $E \otimes R_{\mathfrak{p}}$ is a free $R_{\mathfrak{p}}$-module of rank $m$. Since $\wedge^{i+1}\left(E \otimes R_{\mathfrak{p}}\right)=\wedge^{i+1}(E)$ $\otimes R_{\mathfrak{p}}$, we find that $\wedge^{i+1}(E) \otimes R_{\mathfrak{p}}=0$ if, and only if, $m \leq i$. On the other hand, it follows from theorem 3.1 that $m \leq i$ if, and only if, $e_{0}+e_{1}+\cdots+e_{1} \notin \mathfrak{p}$. Thus, $\wedge^{i+1}(E) \otimes R_{\mathfrak{p}}=0$ is equivalent with $e_{0}+e_{1}+\cdots+e_{i} \notin \mathfrak{p}$. But $\wedge^{i+1}(E)$ $\otimes R_{\mathfrak{p}}=0$ is equivalent with $a\left(\wedge^{i+1}(E)\right) \notin p$, and therefore $a\left(\wedge^{i+1}(E)\right) \subset p$ is equivalent with $e_{0}+\cdots+e_{i} \in \mathfrak{p}$. Now if $a$ is any ideal of $R$, the intersection 
of all prime ideals containing $\mathfrak{a}$ is $\operatorname{rad} a$ so that we have $\operatorname{rad}\left(\mathfrak{a}\left(\wedge^{i+1} E\right)\right)$ $=\operatorname{rad}\left(R\left(e_{0}+\cdots+e_{i}\right)\right)$. But $a\left(\wedge^{i+1} E\right)$, being the annihilator of a finitely generated projective $R$-module, is generated by an idempotent, while $e_{0}+\cdots$ $+\boldsymbol{e}_{i}$ is also an idempotent. It follows from lemma 4.1 that $a\left(\wedge^{i+1}(E)\right)$ is generated by $e_{0}+e_{1}+\cdots+e_{i}$.

Because $\sum_{i=0}^{n-1} e_{i} \neq 1$ and $\sum_{i=0}^{n} e_{i}=1$, we have $\wedge^{n} E \neq 0$ and $\wedge^{n+1} E=0 . \quad$ Also, $\wedge^{n} E$ is faithful if, and only if, its annihilator is 0 , i.e., if and only if $\sum_{i=0}^{n-1} e_{i}$ $=0$. But $\sum_{i=0}^{n-1} e_{i}=1-e_{n}$, so that $\wedge^{n} E$ is faithful is equivalent with $e_{n}=1$. Since the various $e_{i}$ are mutually orthogonal, the latter condition is equivalent with $\varphi(0, E, X)=X^{n}$. It then follows also that each $a\left(\wedge^{i+1} E\right)=0$ for $i \leq n-1$.

Since $e_{0}=\operatorname{det} 0$ we have immediately the following.

Corollary 4.4. E is faithful if, and only if, $\operatorname{det} 0=0$.

A finitely generated projective $R$-module $E$ of $R$-rank $n$ is called orientable if $\wedge^{n} E$ is a free $R$-module of rank one. By theorem 4.3 we have for such a module $\varphi(0, E, X)=X^{n}$. Obviously free modules are orientable.

Proposition 4.5. If $E_{1}$ and $E_{2}$ are orientable, then $E_{1}+E_{2}$ is also. If $F=E_{1}+E_{2}$, with $F$ and $E_{1}$ orientable, then $E_{2}$ is also orientable.

Proof. Suppose $E_{1}$ and $E_{2}$ are orientable with $R$-ranks $n_{1}$ and $n_{2}$, respectively. Then $\wedge^{n_{i}+j} E_{i}=0$, for $j \geq 1$, so that $\wedge^{n_{1}+n_{2}}\left(E_{1}+E_{2}\right)=\wedge^{n_{1}} E_{1} \otimes \wedge^{n_{2}} E_{2}$. Since the $R$-rank of $E_{1}+E_{2}$ is obviously $n_{1}+n_{2}$, it follows that $E_{1}+E_{2}$ is orientable.

Suppose now that $F=E_{1} \dot{+} E_{2}$, with $E_{1}$ orientable of rank $n$ and $F$ orientable of rank $m$. Since $\varphi(0, F, X)=\varphi\left(0, E_{1}, X\right) \varphi\left(0, E_{9}, X\right)$ and $\varphi(0, F, X)$ $=X^{m}$ while $\varphi\left(0, E_{1}, X\right)=X^{n}$, it follows that $\varphi\left(0, E_{2}, X\right)=X^{m-n}$. Thus the $R$-rank of $E_{2}$ is $n-m$ and therefore $\wedge^{m} F=\wedge^{n} E_{1} \otimes \wedge^{m-n} E_{2}$. Since $\wedge^{m} \dot{F}$ and $\wedge^{n} E_{1}$ are both free of rank one, it follows that $\wedge^{m-n} E_{2}$ is also free of rank one and we conclude that $E_{2}$ is orientable.

The proposition just proved shows that the isomorphism classes of orientable modules constitute a subgroup of the projective class group of $R$.

If $\alpha$ is an endomorphism of a module $E$, then $\alpha$ extends to an endomorphism $\bar{\alpha}$ of $\wedge E$ by defining $\bar{\alpha}\left(x_{1} \wedge \cdots \wedge x_{n}\right)=\alpha\left(x_{1}\right) \wedge \cdots \wedge \alpha\left(x_{n}\right)$. Clearly $\bar{\alpha}$ maps each $\wedge^{n} E$ into itself. 
THEOREM 4.6. Let $E$ be an orientable module of R-rank $n$ and $\alpha$ an endomorphism of $E$. Then the restriction of $\bar{\alpha}$ to $\wedge^{n} E$ coincides with multiplication by $\operatorname{det} \alpha$.

Proof. Suppose $E+E_{1}=F$ with $F$ a free module. Let $n_{1}$ be the $R$-rank of $E_{1}$, so that $F$ has rank $n+n_{1}$. Extend $\alpha$ to an endomorphism $\alpha_{1}$ of $F$ by defining $\alpha_{1}$ to be the identity on $E_{1}$; then $\operatorname{det} \alpha=\operatorname{det} \alpha_{1}$.

Now $\wedge^{n+n_{1}} F=\wedge^{n} E \otimes \wedge^{n_{1}} E_{1}$, with all three modules free of rank one. It is clear that $\bar{\alpha}_{1}=\bar{\alpha} \otimes 1$ on $\wedge^{n+n_{1}} F$, while $\bar{\alpha}_{1}$ restricted to $\wedge^{n+n_{1}} F$ coincides with multiplication by $\operatorname{det} \alpha_{1}=\operatorname{det} \alpha$. The result follows immediately.

\section{REFERENCES}

[1] M. Auslander and O. Goldman, Maximal Orders, Trans. Amer. Math. Soc., 97 (1960), pp. 1-24.

[2] G. Birkhoff and S. MacLane, A survey of modern algebra, New York, 1953.

[3] H. Cartan and S. Eilenberg, Homological algebra, Princeton, 1956.

[4] C. Chevalley, Fundamental concepts of algebra, New York, 1956.

Brandeis University 